\title{
The Predictive Value of Systemic Immune-inflammation Index on Bladder Recurrence on Upper Tract Urothelial Carcinoma Outcomes After Radical Nephroureterectomy
}

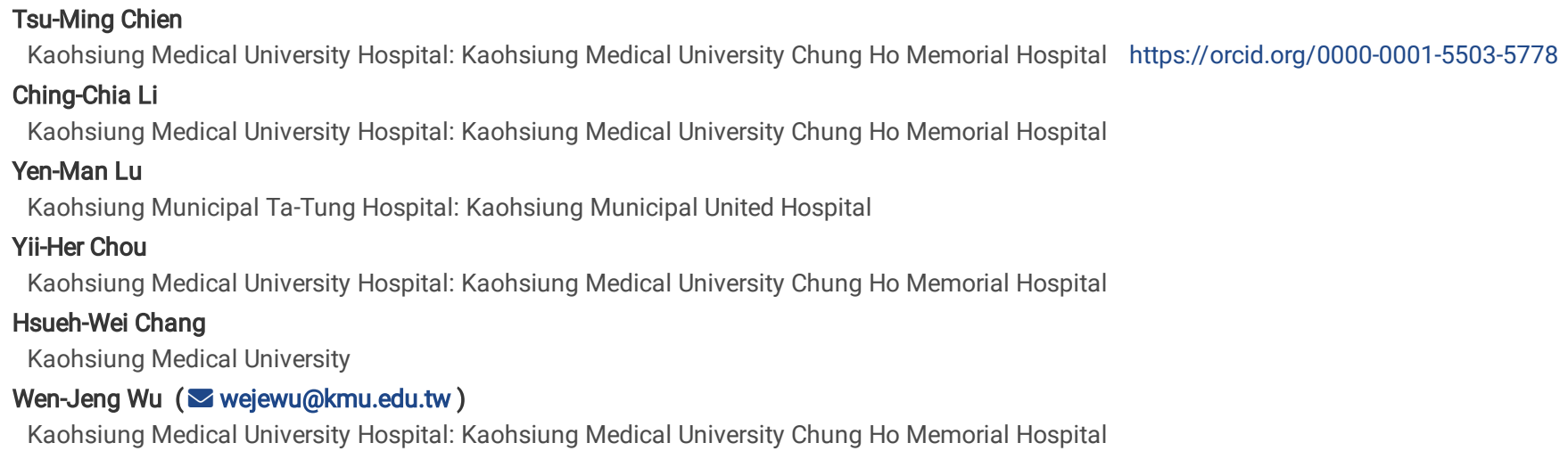

Keywords: Upper tract urothelial carcinoma, bladder recurrence, survival, platelet-lymphocyte, neutrophile-lymphocyte, systemic immune-inflammation index

Posted Date: August 24th, 2021

DOl: https://doi.org/10.21203/rs.3.rs-829449/v1

License: (c) (i) This work is licensed under a Creative Commons Attribution 4.0 International License. Read Full License

Version of Record: A version of this preprint was published at Journal of Clinical Medicine on November 12th, 2021. See the published version at https://doi.org/10.3390/jcm10225273. 


\section{Abstract}

Background: To assess the prognostic significance of pre-treatment lymphocyte-related systemic inflammatory biomarkers in upper tract urothelial carcinoma (UTUC) patients.

Methods: This study included non-metastatic UTUC patients treated at our hospital between 2001 and 2013. The receiver operating characteristic curve was used to obtain the optimal neutrophile-lymphocyte ratio (NLR), platelet-lymphocyte ratio (PLR), and systemic immune-inflammation index (SII). Multivariate logistic regression was performed to investigate the relationship between NLR, PLR, and SII and clinical pathologic characteristics. The Kaplan-Meier method was used to calculate the metastasis-free survival (MFS), cancer-specific survival (CSS), and bladder recurrence-free survival (BRFS) and the log-rank test to compare the survival rate.

Results: Overall, 376 patients were included in the current study. Multivariate analysis revealed that an elevated SIl was associated with advanced pathologic tumor stage, lymph node invasion, and adjuvant chemotherapy; high NLR was associated with older age, hemodialysis status, anemia, multifocal tumor, advanced pathologic tumor stage, and adjuvant chemotherapy; and high PLR was associated with older age, anemia, advanced pathologic tumor stage, and adjuvant chemotherapy. The Kaplan-Meier analysis indicated that patients exhibiting higher NLR, PLR, and SIl showed significantly poor MFS and CSS rates. Only high SIl showed significantly worse BRFS rates.

Conclusions: The NLR, PLR, and SII were independent predictive factors for both MFS and CSS in UTUC patients. Among the factors, only elevated SII can predict bladder recurrence. Therefore, the patients might need close bladder monitoring during the follow-up.

\section{Introduction}

Urothelial carcinoma (UC) is the most common malignancy and includes UC of the urinary bladder (UBUC) and upper tract urothelial carcinoma (UTUC). UTUCs are uncommon, accounting for only $5-10 \%$ of UCs [1], with an estimated annual incidence in Western countries of almost two cases per 100,000 inhabitants [1, 2]. During initial diagnosis, almost $60 \%$ of UTUCs were invasive, which is higher than that in UBUC [3]. Several studies have attempted to identify prognostic factors in patients with UTUC [3-6]. These models are based on age, tumor grade, pathologic tumor grade, lymph node metastasis, lymphovascular invasion, tumor multifocality, and tumor architecture [3-6]. With the development of current genomics studies of UTUC [7], physicians can integrate the clinical pathologic characteristics with genetic and molecular subtyping of UTUC. Knowing the different genotype and phenotype features can lay the groundwork for a deeper understanding of UTUC biology [8]. This information is extremely beneficial in personalized treatment regarding postoperative neoadjuvant chemotherapy and follow-up plan. However, most of these factors are obtained only after surgical removal of the tissue. The considerable improvement of imaging study such as computed tomography or magnetic resonance imaging can provide valuable information in patient counseling and treatment planning [9]; however, its accuracy does not meet the standard for individual treatment decision-making. Therefore, preoperative data to predict recurrence and survival is urgently needed.

Accumulating studies have proved that inflammatory reaction in tumor microenvironment is associated with tumor development and progression [10, 11]. As inflammation can stimulate leukocytosis, neutrophilia, and thrombocytosis, various blood-based inflammatory markers, such as neutrophile-to-lymphocyte ratio (NLR), platelet-to-lymphocyte ratio (PLR), and monocyte-to-lymphocyte ratio (MLR), have been investigated to improve prognostic tools for risk stratification and outcomes in UTUC [12-14]. Recently, a relative new biomarker, the systemic immune-inflammation index (SII), based on neutrophile, lymphocyte, and platelet counts have been developed and showed a high diagnostic value for the prognosis of urologic cancer [15]. The underlying mechanisms for the development of elevated SII in UTUC cancer patients and the subsequent increase in the poor survival of the disease remain unclear. However, these markers with respect to survival might be explained by the relationship among cancers to platelet, neutrophile, and lymphocyte levels. Platelets were reported to be a dangerous alliance of cancer cells and are a close engager in multiple processes of cancer metastasis [16]. Cancer cells can escape from immune cell cytotoxicity by platelet adhesion which forms a protective cloak. Once tumor cells enter the blood, they immediately activate platelets to form a permissive microenvironment. Platelets save tumor cells from shear forces and NK cell assaults and secret chemokines to recruit myeloid cells. This causes the tumor cell platelet embolus to stop at the vascular wall. Then, platelet-derived growth factors confer a mesenchymal-like phenotype to tumor cells and open the capillary endothelium to expedite extravasation in distant organs. Eventually, platelet-secreted growth factors stimulate tumor cell proliferation to micrometastatic foci [17]. Neutrophils, the most abundant white blood cells in the circulation system, constitute a significant part of the tumor microenvironment. Tumor-associated neutrophils secrete cytokines and chemokines to exert an antitumor activity. Furthermore, neutrophiles stimulate immunosuppression, tumor growth, angiogenesis, and metastasis $[18,19]$. Therefore, the elevation of both platelets and neutrophiles stand for the tumor aggressiveness. In contrast, Lymphocytes are known to play an important role in cellular and humoral antitumor immune responses. Activated and proliferating lymphocytes play a role in cytotoxic cell death and inhibit tumor cell proliferation and migration by secreting cytokines such as interferon gamma (IFN- $\mathrm{y}$ ) and tumor necrosis factor alpha (TNF-a). Therefore, low lymphocyte count may reflect the impaired host immunosurveillance and represent an unfavorable prognostic factor for clinical outcomes in patients with tumors [20,21]. Considering these mechanisms, a higher SII representing higher platelet and neutrophile counts and lower lymphocytes may predict poor survival.

The prognostic significance of SII in UTUC or its relative utility when compared with other inflammatory indexes has not been fully investigated. Currently, only a few studies [6, 22-23] had reported the predictive value of the SII as a predictor of UTUC treated by radical nephroureterectomy. In the present study, we retrospectively enrolled 376 patients and investigated the prognostic significance of SII in UTUC patients. Moreover, we compared the clinicopathologic effect on SII value with NLR and PLR.

\section{Methods}


Patients who underwent either open or laparoscopic radical nephroureterectomy (RNU) with bladder cuff excision for non-metastatic UTUC between 2001 and 2013 at Kaohsiung Medical University Hospital were included. The present study was approved by the review board of our institution (KMUH-IRB-20120138). All patients signed the informed consent form. Furthermore, all patients routinely took blood examinations before surgical treatment. Clinical parameters including demographic characteristics, pathological features, oncologic follow-up, and the cause leading to mortality were retrospectively collected. Patients with neoadjuvant chemotherapy or radiotherapy, concurrent muscle-invasive bladder tumor, acute blood disorders, bone marrow diseases, and incomplete clinical information were excluded. We also excluded patients with concurrent bladder tumor. Tumor stage was evaluated according to the 2002 American Joint Committee Cancer TNM system. All cases were reviewed by two pathologists and re-classified as low-or high-grade using the 2004 WHO grading system. Renal function was evaluated with estimated glomerular filtration rate (eGFR) using the Chronic Kidney Disease Epidemiology Collaboration (CKDEPI) creatinine-based formula [15].

\section{Treatment and follow-up}

Patients who underwent either open or laparoscopic RNU with bladder cuff excision for non-metastatic UTUC between 2001 and 2013 at Kaohsiung Medical University Hospital were included. The present study was approved by the review board of our institution (KMUH-IRB-20120138). All patients routinely took blood examinations before surgical treatment. We retrospectively collected data regarding clinical parameters such as demographic characteristics, pathological features, oncologic follow-up, and the cause of death. We also excluded patients with acute blood disorders, bone marrow diseases, concurrent muscle-invasive bladder tumor, neoadjuvant chemotherapy or radiotherapy and incomplete clinical information. Tumor stage was evaluated based on the 2002 American Joint Committee Cancer TNM system. The cases of all patients were reviewed by two pathologists and were re-classified as low- and highgrade based on the 2004 WHO grading system. The Chronic Kidney Disease Epidemiology Collaboration (CKD-EPI) creatinine-based formula [24] was used to evaluate patient renal function.

\section{Treatment and follow-up}

For low-risk tumor, first cystoscopy was arranged at three months after operation. If negative, a subsequent cystoscopy was arranged nine months later. Since the next year, annal cystoscopy was arranged for five years. Patients with high-risk tumor, cystoscopy and urinary cytology were suggested every three months in the first two years and every six months in the next two years. Since the fifth year, yearly follow-ups were arranged. For the first two years, computed tomography (CT) was arranged every six months. After that, yearly CT was suggested. Patients who had advanced pathologic stage, lymph node invasion, and metastatic progression were candidates for adjuvant therapy. After obtaining the patients' willingness and renal function into consideration, 71 cases received systemic chemotherapy and 30 cases received radiation therapy. European association of Urology Guidelines suggest that a single post-operative intravesical instillation of chemotherapy to lowers the bladder cancer recurrence rate [2]. Despite level 1 evidence, only a small minority (42 patients, $11.2 \%$ ) of patients received adjuvant single postoperative instillations of chemotherapy after RNU in our hospital.

\section{Definitions of inflammatory indexes}

The inflammatory indexes were calculated preoperative inflammatory indicators with the following formulas: SII = platelet counts $\mathrm{x}$ neutrophile counts/lymphocyte counts; NLR = neutrophile counts/lymphocyte counts; and PLR = platelet counts/lymphocyte counts. The optimal cut-off value for metastasis-free survival (MFS), cancer-specific survival (CSS), and bladder recurrence-free survival (BRFS) were defined by creating a time-dependent receiver operating characteristic (ROC) curve with MFS, CSS, and BRFS, as the endpoint to yield the highest Youden index value (i.e., sensitivity + specificity - 1), respectively. The Youden index can provide the optimal cut-off values from a continuous variable and off the best for both sensitivity and specificity.

\section{Statistical analysis}

The differences between categorical parameters were assessed using a $\chi^{2}$ or Fisher's exact test. The Kaplan-Meier method was applied to estimate the effect of the three inflammatory indexes SII, NLR, PLR as prognostic factors on (MFS, CSS, and BRFS. Survival rates were recorded from the day of RNU to metastatic progression, cancer-specific death, or the latest visit and bladder recurrence. Specifically, patients who died due to UTUC, including cancer progression and distant metastasis in the follow-up period, were defined to have cancer-specific death. Survival curves were compared using a log-rank test. Univariate and multivariate survival analysis were performed to identify risk factors for prognosis. Only significant prognostic factors in univariate analysis were included in multivariate Cox proportional hazard model to identify independent predictors for MFS, CSS, and BRFS. $P<0.05$ was considered statistically significant. SPSS 20.0 (SPSS Inc., Chicago, IL, USA) was used for all statistical analyses.

\section{Results}

A total of 376 UTUC patients were included in the present study (Table 1). There were 146 (38.8\%) male patients and 230 (61.2\%) female patients. Female were dominant in our study cohort. The mean age was $65.9 \pm 10.8$ years. The mean follow-up time after surgery was $108.6 \pm 39.7$ months. Moreover, 101 patients (26.8\%) had bladder cancer history, 146 patients (38.8\%) had pathologic pTa/pTis/pT1 stage, 68 (23.4\%) had pT2 stage, 114 (30.3\%) pT3 stage, and $28(7.4 \%)$ had pT4 stage. ROC analysis revealed that the optimal cut-off values of SII, NLR, and PLR for MFS and CSS were 460, 3.5, and 162 and 485, 2.9, and 150, respectively. The cut-off values of BRFS were 490, 3.5, and 160. Table 1 using the CSS cut-off values shows that an elevated SII was associated with bladder cancer history, advanced pathologic tumor stage, lymph node invasion, and adjuvant chemotherapy; high NLR was associated with older age, hemodialysis status, anemia, multifocal tumor, advanced pathologic tumor stage, and adjuvant chemotherapy; and high PLR was associated with older age, anemia, advanced pathologic tumor stage, and adjuvant chemotherapy. These three inflammatory indexes were closely linked to each other. 
Table 1

Associations of NLR, PLR, SII and clinicopathologic characteristics in 376 patients treated with radical nephroureterectomy for upper tract urothelial carcinoma.

\begin{tabular}{|c|c|c|c|c|c|c|c|c|c|c|}
\hline \multirow[t]{3}{*}{ Parameters } & \multirow[t]{2}{*}{ All } & \multicolumn{2}{|l|}{ NLR } & \multicolumn{4}{|c|}{ PLR } & \multicolumn{3}{|c|}{ SII } \\
\hline & & $>2.9$ & $\leq 2.9$ & 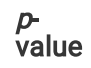 & $>150$ & $\leq 150$ & $\begin{array}{l}p- \\
\text { value }\end{array}$ & $>485$ & $\leq 485$ & $\begin{array}{l}p- \\
\text { value }\end{array}$ \\
\hline & $n(\%)$ & $n(\%)$ & $n(\%)$ & & $\mathrm{n}(\%)$ & $\mathrm{n}(\%)$ & & $\mathrm{n}(\%)$ & $\mathrm{n}(\%)$ & \\
\hline Age & & & & 0.006 & & & $<.001$ & & & 0.972 \\
\hline$\leq 70$ & $208(55.3)$ & $70(46.7)$ & 138(61.1) & & 28(35.9) & $180(60.4)$ & & $116(55.2)$ & $92(55.4)$ & \\
\hline$>70$ & 168(44.7) & $80(53.1)$ & $88(38.9)$ & & $50(64.2)$ & 118(39.6) & & $94(44.8)$ & $74(44.6)$ & \\
\hline Gender & & & & 0.214 & & & 0.263 & & & 0.601 \\
\hline Male & 146(38.8) & $64(42.7)$ & $82(36.3)$ & & 26(33.3) & $120(40.3)$ & & $84(40.0)$ & $62(37.3)$ & \\
\hline Female & $230(61.2)$ & $86(57.3)$ & 144(63.7) & & $52(66.7)$ & 178(59.7) & & $126(60.0)$ & $104(62.7)$ & \\
\hline Smoking & & & & 0.182 & & & 0.175 & & & 0.104 \\
\hline No & $308(81.9)$ & 118(78.7) & 190(84.1) & & $68(87.2)$ & $240(80.5)$ & & 166(79.0) & $142(85.5)$ & \\
\hline Yes & $68(18.1)$ & $32(21.3)$ & $36(15.9)$ & & $10(12.8)$ & $58(19.5)$ & & $44(21.0)$ & $24(14.5)$ & \\
\hline Bladder cancer history & & & & 0.263 & & & 0.989 & & & 0.025 \\
\hline No & $275(73.1)$ & $105(70.0)$ & 170(75.2) & & $57(73.1)$ & 218(73.2) & & 144(68.6) & 131(78.9) & \\
\hline Yes & 101(26.9) & $45(30.0)$ & $56(24.8)$ & & $21(26.9)$ & $80(26.8)$ & & $66(31.4)$ & $35(21.1)$ & \\
\hline $\mathrm{BMI}$ & & & & 0.056 & & & 0.096 & & & 0.462 \\
\hline$>27$ & $62(16.5)$ & 18(12.0) & $44(19.5)$ & & $8(10.3)$ & $54(18.1)$ & & $32(15.2)$ & $30(18.1)$ & \\
\hline$\leq 27$ & $314(83.5)$ & 132(88.0) & 182(80.5) & & 70(89.7) & 244(81.9) & & 178(84.8) & 136(81.9) & \\
\hline ESRD & & & & 0.044 & & & 0.479 & & & 0.151 \\
\hline No & $300(79.8)$ & $112(74.7)$ & 188(83.2) & & $60(76.9)$ & $240(80.5)$ & & 162(77.1) & 138(83.1) & \\
\hline Yes & $76(20.2)$ & $38(25.3)$ & $38(16.8)$ & & 18(23.1) & $58(19.5)$ & & $48(22.9)$ & $28(16.9)$ & \\
\hline Anemia & & & & 0.016 & & & $<.001$ & & & 0.127 \\
\hline No & 138(36.7) & $44(29.3)$ & $94(41.6)$ & & 14(17.9) & $124(41.6)$ & & 70(33.3) & $68(41.0)$ & \\
\hline Yes & $238(63.3)$ & 106(70.7) & $132(58.4)$ & & $64(82.1)$ & $174(58.4)$ & & $140(66.7)$ & $98(59.0)$ & \\
\hline ECOG score & & & & 0.156 & & & 0.290 & & & 0.080 \\
\hline 0,1 & 297(79.0) & 113(75.3) & 184(81.4) & & 65(83.3) & 232(77.9) & & 159(75.7) & 138(83.1) & \\
\hline 2,3 & $79(21.0)$ & $37(24.7)$ & $42(18.6)$ & & 13(16.7) & $66(22.1)$ & & $51(24.3)$ & $28(16.9)$ & \\
\hline CKD stage & & & & 0.053 & & & 0.223 & & & 0.699 \\
\hline Stage 1 & $36(9.6)$ & $6(4.0)$ & $30(13.3)$ & & $4(5.1)$ & $32(10.7)$ & & 18(8.6) & 18(10.8) & \\
\hline Stage 2 & $78(20.7)$ & $32(21.3)$ & $46(20.4)$ & & 18(23.1) & $60(20.1)$ & & $44(21.0)$ & $34(20.5)$ & \\
\hline Stage 3 & $154(41.0)$ & $64(42.7)$ & $90(39.8)$ & & $28(35.9)$ & $126(42.3)$ & & $84(40.0)$ & $70(42.2)$ & \\
\hline Stage 4 & $24(6.4)$ & $10(6.7)$ & $14(6.2)$ & & $8(10.3)$ & $16(5.4)$ & & $12(5.7)$ & $12(7.2)$ & \\
\hline Stage 5 & $84(22.3)$ & $38(25.3)$ & $46(20.4)$ & & $20(25.6)$ & $64(21.5)$ & & $52(24.8)$ & $32(19.3)$ & \\
\hline $\begin{array}{l}\text { Advanced CKD stage (Stage 4, } \\
\text { 5) }\end{array}$ & 108(28.7) & $48(32.0)$ & $60(26.5)$ & 0.253 & $28(35.9)$ & $80(26.8)$ & 0.116 & $64(30.5)$ & $44(26.5)$ & 0.398 \\
\hline Type of operation & & & & 0.304 & & & 0.712 & & & 0.093 \\
\hline Open & $244(64.9)$ & $48(32.0)$ & $84(37.2)$ & & $52(66.7)$ & 192(66.4) & & $144(68.6)$ & $100(60.2)$ & \\
\hline Laparoscopic & 132(35.1) & $102(68.0)$ & $142(62.8)$ & & $26(33.3)$ & 106(35.6) & & $66(31.4)$ & $66(39.8)$ & \\
\hline Tumor location & & & & 0.910 & & & 0.131 & & & 0.445 \\
\hline Pyelocaliceal & $170(45.2)$ & $68(45.3)$ & $102(45.1)$ & & $42(53.8)$ & $128(43.0)$ & & $98(46.7)$ & $72(43.4)$ & \\
\hline Ureteral & $132(35.1)$ & $54(36.0)$ & $78(34.5)$ & & 26(33.3) & 106(35.6) & & $68(32.4)$ & $64(38.6)$ & \\
\hline
\end{tabular}




\begin{tabular}{|c|c|c|c|c|c|c|c|c|c|c|}
\hline \multirow[t]{2}{*}{ Parameters } & \multirow[t]{2}{*}{ All } & \multicolumn{2}{|l|}{ NLR } & \multicolumn{4}{|c|}{ PLR } & \multicolumn{3}{|c|}{ SII } \\
\hline & & $>2.9$ & $\leq 2.9$ & $\begin{array}{l}p- \\
\text { value }\end{array}$ & $>150$ & $\leq 150$ & $\begin{array}{l}p- \\
\text { value }\end{array}$ & $>485$ & $\leq 485$ & $\underset{\text { value }}{p}$ \\
\hline Both & 74(19.7) & $28(18.7)$ & $46(20.4)$ & & $10(12.8)$ & $64(21.5)$ & & $44(21.0)$ & $30(18.1)$ & \\
\hline Multifocality & & & & 0.021 & & & 0.568 & & & 0.099 \\
\hline Single & 298(79.3) & 110(73.3) & 188(83.2) & & $60(76.9)$ & 238(79.9) & & $160(76.2)$ & 138(83.1) & \\
\hline Multiple & 78(20.7) & $40(26.7)$ & $38(16.8)$ & & $18(23.1)$ & $60(20.1)$ & & $50(23.8)$ & $28(16.9)$ & \\
\hline Pathologic T stage & & & & 0.001 & & & 0.018 & & & 0.017 \\
\hline $\mathrm{pTa} / \mathrm{pTis} / \mathrm{pT} 1$ & $146(38.8)$ & $46(30.7)$ & $100(44.2)$ & & $26(33.3)$ & $120(40.3)$ & & $70(33.3)$ & $76(45.8)$ & \\
\hline pT2 & $68(23.4)$ & $38(25.3)$ & $50(22.1)$ & & $20(25.6)$ & $68(22.8)$ & & $52(24.8)$ & $36(21.7)$ & \\
\hline рT3 & $114(30.3)$ & $46(30.7)$ & $68(30.1)$ & & $20(25.6)$ & $94(31.5)$ & & $66(31.4)$ & $48(28.9)$ & \\
\hline pT4 & $28(7.4)$ & $20(13.3)$ & $8(3.5)$ & & $12(15.4)$ & $16(5.4)$ & & $22(10.5)$ & $6(3.6)$ & \\
\hline Advanced pT stage (pT3, pT4) & & & & 0.042 & & & 0.505 & & & 0.063 \\
\hline No & $234(62.2)$ & $84(56.0)$ & $150(66.4)$ & & $46(59.0)$ & $188(63.1)$ & & $122(58.1)$ & $112(67.5)$ & \\
\hline Yes & 142(37.8) & $66(44.0)$ & $76(33.6)$ & & $32(41.0)$ & $110(36.9)$ & & $88(41.9)$ & $54(32.5)$ & \\
\hline Pathologic $\mathrm{N}$ stage & & & & 0.659 & & & 0.479 & & & 0.003 \\
\hline pNO & 68(18.1) & $23(15.3)$ & $36(15.9)$ & & $22(22.4)$ & $42(14.1)$ & & $31(14.8)$ & $35(21.1)$ & \\
\hline $\mathrm{pNx}$ & $232(61.7)$ & $95(63.3)$ & $146(64.6)$ & & $58(59.2)$ & 198(66.4) & & $125(59.5)$ & $109(65.7)$ & \\
\hline $\mathrm{pN+}$ & $76(20.2)$ & $32(21.3)$ & $44(19.5)$ & & $18(23.1)$ & $58(19.5)$ & & $54(25.7)$ & $22(13.3)$ & \\
\hline Grade & & & & 0.257 & & & 0.941 & & & 0.527 \\
\hline Low & $76(20.2)$ & $26(17.3)$ & $50(22.1)$ & & $16(20.5)$ & $60(20.1)$ & & $40(19.0)$ & $36(21.7)$ & \\
\hline High & $300(79.8)$ & $124(82.7)$ & $176(77.9)$ & & $62(79.5)$ & $238(79.9)$ & & $170(81.0)$ & $130(78.3)$ & \\
\hline Adjuvant chemotherapy & & & & 0.015 & & & 0.009 & & & $\dot{0}_{0.001}$ \\
\hline No & $308(81.9)$ & $114(76.0)$ & 194(85.8) & & $56(71.8)$ & $252(84.6)$ & & $158(75.2)$ & 150(90.4) & \\
\hline Yes & $68(18.1)$ & $36(24.0)$ & $32(14.2)$ & & $22(28.2)$ & $46(15.4)$ & & $52(24.8)$ & $16(9.6)$ & \\
\hline NLR & & & & & & & $\begin{array}{l}< \\
0.001\end{array}$ & & & $\dot{ }_{0.001}$ \\
\hline$>2.9$ & - & - & - & & 76(97.4) & $74(24.8)$ & & $140(66.7)$ & $10(6.0)$ & \\
\hline$\leq 2.9$ & - & - & - & & $2(2.6)$ & $224(75.2)$ & & $70(33.3)$ & 156(94.0) & \\
\hline PLR & & & & $\begin{array}{l}< \\
0.001\end{array}$ & & & & & & $\dot{ }_{0.001}$ \\
\hline$>150$ & 78(20.7) & $76(50.7)$ & $2(0.9)$ & & - & - & & $78(37.1)$ & $0(0.0)$ & \\
\hline$\leq 150$ & $298(79.3)$ & $74(49.3)$ & $224(99.1)$ & & - & - & & $132(62.9)$ & $166(100.0)$ & \\
\hline SII & & & & $\begin{array}{l}< \\
0.001\end{array}$ & & & $\stackrel{<}{0.001}^{-}$ & & & \\
\hline$>485$ & $210(55.9)$ & 140(93.3) & $70(31.0)$ & & $78(100.0)$ & $132(44.3)$ & & - & - & \\
\hline$\leq 485$ & $166(44.1)$ & $10(6.7)$ & $156(69.0)$ & & $0(0.0)$ & $166(55.7)$ & & - & - & \\
\hline
\end{tabular}

Kaplan-Meier analysis for MFS, CSS, and BRFS

Ninety-four patients (25.0\%) from our study cohort experienced metastasis events (female, 58 patients; male, 36 patients). The 3- and 5-year MFS rates were $76.6 \%$ and $71.8 \%$, respectively. Univariate analysis showed that bladder cancer history $(p=0.004)$, advanced CKD stage $(p=0.004)$, ureteral tumors $(p=0.029)$, advanced pathological stage $(p<0.001)$, multifocal tumor $(p=0.013)$ lymph node invasion $(p<0.001)$, high tumor grade $(p<0.001)$, adjuvant chemotherapy $(p<0.001)$, elevated NLR $(p<0.001)$, elevated PLR $(p<0.001)$, and elevated SII $(p<0.001)$ were associated with worse MFS (Table 2). Ureteral tumors $(p=$ $0.019)$, advanced pathological tumor stage $(p<0.001)$, high tumor grade $(p=0.039)$, adjuvant chemotherapy $(p<0.001)$, elevated NLR $(p=0.021)$, elevated 
$\operatorname{PLR}(p=0.009)$, and elevated SII $(p=0.004)$ were independent risk factors for lower MFS in multivariate analysis. The Kaplan-Meier analysis indicated that patients exhibiting higher NLR, PLR, and SII showed significantly poor MFS rates (Figs. 1a, 1b, and 1c; log-rank, all $P<0.001$ ). 
Table 2

Univariate and multivariate analyses predicting CSS, MFS and BRFS in patients $(n=376)$ with UTUC after RNU

\begin{tabular}{|c|c|c|c|c|c|c|c|c|c|c|c|c|}
\hline Parameters & MFS & & & & css & & & & BRFS & & & \\
\hline & Univariate & $p$ & Multivariate & $p$ & Univariate & $p$ & Multivariate & $p$ & Univariate & $p$ & Multivariate & $p$ \\
\hline & Analysis & & analysis & & analysis & & analysis & & analysis & & analysis & \\
\hline & $\mathrm{HR}(95 \% \mathrm{Cl})$ & & $\mathrm{HR}(95 \% \mathrm{Cl})$ & & $\mathrm{HR}(95 \% \mathrm{Cl})$ & & $\mathrm{HR}(95 \% \mathrm{Cl})$ & & $\mathrm{HR}(95 \% \mathrm{Cl})$ & & $\mathrm{HR}(95 \% \mathrm{Cl})$ & \\
\hline \multicolumn{13}{|l|}{ Age (Years) } \\
\hline Over 70 years & $\begin{array}{l}1.1(0.7- \\
1.8)\end{array}$ & 0.632 & & & $\begin{array}{l}1.3(0.7- \\
2.2)\end{array}$ & 0.366 & & & $\begin{array}{l}1.2(0.8- \\
1.9)\end{array}$ & 0.391 & & \\
\hline \multicolumn{13}{|l|}{ Gender } \\
\hline $\begin{array}{l}\text { Male vs } \\
\text { Female }\end{array}$ & $\begin{array}{l}1.0(0.9- \\
1.2)\end{array}$ & 0.509 & & & $\begin{array}{l}1.2(0.9- \\
1.6)\end{array}$ & 0.329 & & & $\begin{array}{l}2.2(1.4- \\
3.4)\end{array}$ & 0.001 & $2.4(1.5-4.0)$ & 0.00 \\
\hline \multicolumn{13}{|l|}{ Smoking } \\
\hline Yes vs No & $\begin{array}{l}1.3(0.8- \\
2.1)\end{array}$ & 0.352 & & & $\begin{array}{l}1.2(0.6- \\
2.3)\end{array}$ & 0.674 & & & $\begin{array}{l}1.2(0.7- \\
2.2)\end{array}$ & 0.465 & & \\
\hline \multicolumn{13}{|l|}{$\begin{array}{l}\text { Bladder } \\
\text { cancer history }\end{array}$} \\
\hline Yes vs No & $\begin{array}{l}1.3(1.1- \\
1.5)\end{array}$ & 0.004 & $\begin{array}{l}1.2(0.9- \\
1.4)\end{array}$ & 0.586 & $\begin{array}{l}1.2(0.9- \\
1.5)\end{array}$ & 0.126 & & & $\begin{array}{l}1.8(1.5- \\
2.1)\end{array}$ & <. 0.001 & $\begin{array}{l}1.6(1.4- \\
1.9)\end{array}$ & $<.00$ \\
\hline \multicolumn{13}{|l|}{$\mathrm{BMI}\left(\mathrm{Kg} / \mathrm{m}^{2}\right)$} \\
\hline $\begin{array}{l}\text { Over } 27 \\
\mathrm{Kg} / \mathrm{m}^{2}\end{array}$ & $\begin{array}{l}1.7(0.9- \\
3.0)\end{array}$ & 0.078 & & & $\begin{array}{l}2.8(1.0- \\
7.3)\end{array}$ & 0.025 & $\begin{array}{l}6.1(1.7- \\
21.1)\end{array}$ & 0.005 & $\begin{array}{l}1.1(0.7- \\
1.8)\end{array}$ & 0.579 & & \\
\hline \multicolumn{13}{|l|}{ ESRD } \\
\hline Yes vs No & $\begin{array}{l}1.4(0.9- \\
2.4)\end{array}$ & 0.138 & & & $\begin{array}{l}1.0(0.6- \\
1.8)\end{array}$ & 0.964 & & & $\begin{array}{l}1.6(0.9- \\
2.7)\end{array}$ & 0.080 & & \\
\hline \multicolumn{13}{|l|}{ Anemia } \\
\hline Yes vs No & $\begin{array}{l}1.1(0.7- \\
1.5)\end{array}$ & 0.711 & & & $\begin{array}{l}1.0(0.6- \\
1.8)\end{array}$ & 0.995 & & & $\begin{array}{l}1.2(0.9- \\
1.8)\end{array}$ & 0.132 & & \\
\hline \multicolumn{13}{|l|}{ ECOG } \\
\hline 2,3 vs 0,1 & $\begin{array}{l}1.5(0.9- \\
2.6)\end{array}$ & 0.125 & & & $\begin{array}{l}1.5(0.8- \\
2.8)\end{array}$ & 0.241 & & & $\begin{array}{l}1.2(0.7- \\
2.0)\end{array}$ & 0.518 & & \\
\hline \multicolumn{13}{|l|}{ CKD stage } \\
\hline $\begin{array}{l}\text { Stage } 2 \text { vs } \\
\text { Stage } 0,1\end{array}$ & $\begin{array}{l}3.9(0.4- \\
34.1)\end{array}$ & 0.199 & & & $\begin{array}{l}1.1(0.4- \\
3.4)\end{array}$ & 0.890 & & & $\begin{array}{l}1.4(0.5- \\
3.5)\end{array}$ & 0.500 & & \\
\hline $\begin{array}{l}\text { Stage } 3 \text { vs } \\
\text { Stage } 0,1\end{array}$ & $\begin{array}{l}4.1(0.5- \\
34.7)\end{array}$ & 0.167 & & & $\begin{array}{l}2.4(0.8- \\
7.4)\end{array}$ & 0.104 & & & $\begin{array}{l}1.4(0.6- \\
3.3)\end{array}$ & 0.442 & & \\
\hline $\begin{array}{l}\text { Stage } 4 \text { vs } \\
\text { Stage } 0,1\end{array}$ & $\begin{array}{l}6.9(0.7- \\
71.7)\end{array}$ & 0.083 & & & $\begin{array}{l}1.6(0.4- \\
7.1)\end{array}$ & 0.535 & & & $\begin{array}{l}1.2(0.3- \\
3.9)\end{array}$ & 0.803 & & \\
\hline $\begin{array}{l}\text { Stage } 5 \text { vs } \\
\text { Stage } 0,1\end{array}$ & $\begin{array}{l}1.1(0.1- \\
13.7)\end{array}$ & 0.960 & & & $\begin{array}{l}1.2(0.4- \\
3.6)\end{array}$ & 0.791 & & & $\begin{array}{l}1.8(0.7- \\
4.3)\end{array}$ & 0.224 & & \\
\hline \multicolumn{13}{|l|}{$\begin{array}{l}\text { Advanced } \\
\text { CKD (Stage 4, } \\
\text { 5) }\end{array}$} \\
\hline Yes vs No & $\begin{array}{l}2.0(1.2- \\
3.2)\end{array}$ & 0.004 & $\begin{array}{l}1.2(0.5- \\
2.7)^{-}\end{array}$ & 0.686 & $\begin{array}{l}1.6(0.9- \\
2.9)^{-}\end{array}$ & 0.103 & & & $\begin{array}{l}1.2(0.7- \\
2.0)\end{array}$ & 0.453 & & \\
\hline \multicolumn{13}{|l|}{$\begin{array}{l}\text { Type of } \\
\text { operation }\end{array}$} \\
\hline $\begin{array}{l}\text { Laparoscopic } \\
\text { vs open }\end{array}$ & $\begin{array}{l}0.9(0.6- \\
1.5)\end{array}$ & 0.803 & & & $\begin{array}{l}0.6(0.3- \\
1.2)\end{array}$ & 0.135 & & & $\begin{array}{l}0.7(0.4- \\
1.1)\end{array}$ & 0.158 & & \\
\hline \multicolumn{13}{|l|}{$\begin{array}{l}\text { Tumor } \\
\text { location }\end{array}$} \\
\hline $\begin{array}{l}\text { Ureteral vs } \\
\text { Pyelocaliceal }\end{array}$ & $\begin{array}{l}2.5(1.1- \\
5.7)\end{array}$ & 0.029 & $\begin{array}{l}4.2(1.3- \\
13.7)\end{array}$ & 0.019 & $\begin{array}{l}1.2(0.7- \\
2.3)\end{array}$ & 0.058 & & & $\begin{array}{l}1.0(0.6- \\
1.7)\end{array}$ & 0.967 & & \\
\hline $\begin{array}{l}\text { Both vs } \\
\text { Ureteral }\end{array}$ & $\begin{array}{l}1.5(0.5- \\
4.4)\end{array}$ & 0.318 & $\begin{array}{l}1.2(0.6- \\
2.6)\end{array}$ & 0.211 & $\begin{array}{l}1.2(0.6- \\
2.5)\end{array}$ & 0.683 & & & $\begin{array}{l}1.4(0.8- \\
2.7)\end{array}$ & 0.238 & & \\
\hline
\end{tabular}




\begin{tabular}{|c|c|c|c|c|c|c|c|c|c|c|c|c|}
\hline Parameters & MFS & & & & css & & & & BRFS & & & \\
\hline $\begin{array}{l}\text { Both vs } \\
\text { Pyelocaliceal }\end{array}$ & $\begin{array}{l}1.6(0.6- \\
4.2)\end{array}$ & 0.430 & $2.8(0.8-9.0)$ & 0.095 & $\begin{array}{l}1.4(0.7- \\
2.9)\end{array}$ & 0.342 & & & $\begin{array}{l}1.5(0.8- \\
2.6)\end{array}$ & 0.204 & & \\
\hline \multicolumn{13}{|l|}{ Multifocality } \\
\hline $\begin{array}{l}\text { Multiple vs } \\
\text { Single }\end{array}$ & $\begin{array}{l}2.0(1.2- \\
3.4)\end{array}$ & 0.013 & $\begin{array}{l}1.6(0.7- \\
3.9)\end{array}$ & 0.285 & $\begin{array}{l}1.5(0.8- \\
2.8)\end{array}$ & 0.217 & & & $\begin{array}{l}1.1(0.7- \\
1.9)\end{array}$ & 0.654 & & \\
\hline \multicolumn{13}{|l|}{$\begin{array}{l}\text { Pathologic } T \\
\text { stage }\end{array}$} \\
\hline $\begin{array}{l}\text { pT2 vs } \\
\text { pTa/pTis/pT1 }\end{array}$ & $\begin{array}{l}3.0(1.3- \\
7.0)\end{array}$ & 0.008 & $\begin{array}{l}1.2(0.2- \\
6.8)\end{array}$ & 0.185 & $\begin{array}{l}1.7(0.6- \\
4.8)\end{array}$ & 0.289 & $\begin{array}{l}1.4(0.4- \\
4.1)\end{array}$ & 0.593 & $\begin{array}{l}1.0(0.6- \\
1.7)\end{array}$ & 0.924 & $\begin{array}{l}1.2(0.6- \\
2.2)\end{array}$ & 0.68 \\
\hline $\begin{array}{l}\text { pT3 vs } \\
\text { pTa/pTis/pT1 }\end{array}$ & $\begin{array}{l}12.2(5.8- \\
25.7)\end{array}$ & $\begin{array}{l}< \\
0.001\end{array}$ & $\begin{array}{l}1.8(0.9- \\
3.5)\end{array}$ & 0.177 & $\begin{array}{l}7.3(3.2- \\
16.6)\end{array}$ & $\begin{array}{l}< \\
0.001\end{array}$ & $\begin{array}{l}2.2(1.4- \\
3.5)\end{array}$ & 0.001 & $\begin{array}{l}1.1(0.6- \\
1.8)\end{array}$ & 0.803 & $\begin{array}{l}1.4(0.9- \\
2.1)\end{array}$ & 0.13 \\
\hline $\begin{array}{l}\text { pT4 vs } \\
\text { pTa/pTis/pT1 }\end{array}$ & $\begin{array}{l}13.6(5.1- \\
36.3)\end{array}$ & $<.001$ & $\begin{array}{l}2.6(1.5- \\
4.7)\end{array}$ & $\begin{array}{l}< \\
0.001\end{array}$ & $\begin{array}{l}9.6(3.3- \\
27.4)\end{array}$ & $<.001$ & $\begin{array}{l}1.6(1.1- \\
2.4)\end{array}$ & 0.032 & $\begin{array}{l}4.2(1.1- \\
16.4)\end{array}$ & 0.011 & $\begin{array}{l}1.8(1.1- \\
3.2)\end{array}$ & 0.03 \\
\hline \multicolumn{13}{|l|}{$\begin{array}{l}\text { Pathologic N } \\
\text { stage }\end{array}$} \\
\hline $\begin{array}{l}\mathrm{pN}+\mathrm{vs} \\
\mathrm{pN} 0 / \mathrm{pNx}\end{array}$ & $\begin{array}{l}3.8(2.2- \\
6.4)\end{array}$ & $<.001$ & $\begin{array}{l}1.2(0.5- \\
2.9)\end{array}$ & 0.640 & $\begin{array}{l}2.3(1.3- \\
4.3)\end{array}$ & 0.006 & $1.3(0.6-3.0)$ & 0.467 & $\begin{array}{l}1.1(0.7- \\
1.7)\end{array}$ & 0.604 & & \\
\hline \multicolumn{13}{|l|}{ Grade } \\
\hline High vs Low & $\begin{array}{l}5.7(2.2- \\
15.0)\end{array}$ & $<.001$ & $\begin{array}{l}4.4(1.1- \\
17.9)\end{array}$ & 0.039 & $\begin{array}{l}2.3(1.3- \\
4.2)\end{array}$ & 0.032 & $\begin{array}{l}1.1(0.4- \\
3.6)\end{array}$ & 0.830 & $\begin{array}{l}1.9(1.1- \\
3.1)\end{array}$ & 0.020 & $\begin{array}{l}2.5(1.4- \\
4.4)\end{array}$ & 0.01 \\
\hline \multicolumn{13}{|l|}{$\begin{array}{l}\text { Adjuvant } \\
\text { chemotherapy }\end{array}$} \\
\hline Yes vs No & $\begin{array}{l}43.8(20.6- \\
93.3)\end{array}$ & $\begin{array}{l}< \\
0.001\end{array}$ & $\begin{array}{l}40.7(16.3- \\
101.7)\end{array}$ & $\begin{array}{l}< \\
0.001\end{array}$ & $\begin{array}{l}8.9(4.8- \\
16.4)\end{array}$ & $<.001$ & $\begin{array}{l}6.6(3.2- \\
13.7)\end{array}$ & $\begin{array}{l}<.001 \\
0.00\end{array}$ & $\begin{array}{l}4.3(2.5- \\
7.4)\end{array}$ & $<.001$ & $\begin{array}{l}4.8(2.7- \\
8.5)\end{array}$ & $<$ \\
\hline \multicolumn{13}{|l|}{$\begin{array}{l}\text { Post-operative } \\
\text { installation } \\
\text { chemotherapy }\end{array}$} \\
\hline Yes vs No & $\begin{array}{l}0.9(0.8- \\
1.2)\end{array}$ & 0.785 & & & $\begin{array}{l}1.0(0.6- \\
1.3)\end{array}$ & 0.788 & & & $\begin{array}{l}1.1(1.0- \\
1.2)\end{array}$ & 0.028 & $\begin{array}{l}1.0(0.9- \\
1.1)\end{array}$ & 0.67 \\
\hline \multirow[t]{2}{*}{ NLR } & $\begin{array}{l}(>3.5 \text { vs } \leq \\
3.5)\end{array}$ & & & & $\begin{array}{l}(>2.9 \text { vs } \\
\leq 2.9)\end{array}$ & & & & $\begin{array}{l}(>3.5 \text { vs } \\
\leq 3.5)\end{array}$ & & & \\
\hline & $\begin{array}{l}2.3(1.4- \\
3.7)\end{array}$ & $<.001$ & $\begin{array}{l}2.2(1.1- \\
4.3)\end{array}$ & 0.021 & $\begin{array}{l}2.7(1.5- \\
4.7)\end{array}$ & $<.001$ & $\begin{array}{l}2.3(1.2- \\
4.3))\end{array}$ & 0.009 & $\begin{array}{l}1.3(0.8- \\
2.0)\end{array}$ & 0.253 & & \\
\hline \multirow[t]{2}{*}{ PLR } & $\begin{array}{l}(>162 \text { vs } \\
\leq 162)\end{array}$ & & & & $\begin{array}{l}(>150 \text { vs } \\
\leq 150)\end{array}$ & & & & $\begin{array}{l}(>160 \text { vs } \\
\leq 160)\end{array}$ & & & \\
\hline & $\begin{array}{l}3.1(1.8- \\
5.2)\end{array}$ & $<.001$ & $\begin{array}{l}3.6(1.4- \\
9.4)\end{array}$ & 0.009 & $\begin{array}{l}2.2(1.2- \\
4.1)\end{array}$ & 0.009 & $2.0(1.1-4.0)$ & 0.046 & $\begin{array}{l}1.0(0.7- \\
1.5)\end{array}$ & 0.910 & & \\
\hline \multirow[t]{2}{*}{ SII } & $\begin{array}{l}(>460 \text { vs } \\
\leq 460)\end{array}$ & & & & $\begin{array}{l}(>485 \text { vs } \\
\leq 485)\end{array}$ & & & & $\begin{array}{l}(>490 \text { vs } \\
\leq 490)\end{array}$ & & & \\
\hline & $\begin{array}{l}3.4(2.0- \\
5.8)\end{array}$ & $\begin{array}{l}< \\
0.001\end{array}$ & $\begin{array}{l}2.9(1.4- \\
6.1)\end{array}$ & 0.004 & $\begin{array}{l}3.8(2.0- \\
7.4)\end{array}$ & $\begin{array}{l}< \\
0.001\end{array}$ & $\begin{array}{l}3.3(1.3- \\
8.5)\end{array}$ & 0.014 & $\begin{array}{l}1.7(1.1- \\
2.7)\end{array}$ & 0.026 & $\begin{array}{l}1.7(1.1- \\
2.8)\end{array}$ & 0.01 \\
\hline
\end{tabular}

CKD chronic kidney disease; ECOG Eastern Cooperative Oncology Group; ESRD end stage renal disease; NLR neutrophile-lymphocyte ratio; PLRPlateletlymphocyte ratio; SII systemic immune-inflammation index

Sixty patients (15.9\%) had cancer-specific mortality during follow-up (female, 38 patients; male, 22 patients). The 3-and 5-year CSS rates were $84.1 \%$ and $79.2 \%$, respectively. Univariate analysis showed that obesity $(p=0.025)$, advanced pathological stage $(p<0.001)$, lymph node invasion $(p=0.006)$, high tumor grade $(p=0.032)$, adjuvant chemotherapy $(p<0.001)$, elevated NLR $(p<0.001)$, elevated PLR $(p=0.009)$, and elevated SII ( $p<0.001)$ were associated with worse CSS (Table 2). Obsity ( $p=0.005)$, advanced pathological tumor stage $(p<0.001)$, adjuvant chemotherapy $(p<0.001)$, elevated NLR $(p=0.009)$, elevated $\operatorname{PLR}(p=0.046)$, and elevated SII $(p=0.014)$ were independent risk factors for lower CSS in multivariate analysis. Similarly, the Kaplan-Meier analysis revealed that patients exhibiting higher NLR, PLR, and SIl showed significantly worse CSS rates (Figs. 2a, 2b, and 3c).

Additionally, 108 patients (28.7\%) had bladder recurrence during follow-up (female, 52 patients; male, 56 patients). The 3-and 5-year BRFS rates were 78.6\% and $63.5 \%$, respectively. Univariate analysis showed that male gender $(p=0.001)$, bladder cancer history $(p=0.001)$, pT4 pathological stage $(p=0.011)$, high tumor grade $(p=0.020)$, adjuvant chemotherapy $(p<0.001)$, and elevated SII $(p=0.026)$ were associated with worse BRFS (Table 2). Male gender $(p=0.001)$, bladder cancer history $(p<0.001)$, pT4 pathological stage $(p=0.032)$, high tumor grade $(p=0.010)$, adjuvant chemotherapy $(p<0.001)$ and elevated SII $(p=$ 0.016) were independent risk factors for lower BRFS in multivariate analysis. Adjuvant single postoperative instillations of chemotherapy after RNU can 
decrease the bladder recurrence rate in univariate analysis, but fail to achieve statistic difference in multivariate analysis. Further, the Kaplan-Meier analysis indicated that patients with higher SII showed significantly worse BRFS rates (Fig. 3c). Higher NLR and PLR were not associated with BRFS (Fig. 3a, and 3b).

\section{Discussion}

To our knowledge, this is the first study that investigated the prognostic significance of SII on BRFS in UTUC patients. Moreover, we compared the significance of preoperative NLR and PLR with SII in UTUC patients. We demonstrated that elevated SIl was not only associated with worse oncologic outcomes but also predicted bladder recurrence. Our study provides potentially crucial clinical implications. By performing the simple routine preoperative blood examinations, we can identify patients who have higher risk for poor survival. SII was a superior prognostic predictor of bladder recurrence than NLR and PLR. In the current study cohort, elevated SII was noted in $56 \%$ of patients and one-third of these patients suffered from bladder recurrence. A careful postoperative follow-up should be performed in these patients.

A recent meta-analysis [15] analyzed the prognostic value of SII in urologic cancer. It included 14 published papers with 3074 patients and showed that UC patients with high SII had worse prognosis for overall survival. Furthermore, it concluded that SII could be a simple and cost-effective prognostic indicator. In UTUC, three previous studies have investigated the prognostic significance of SII. Jan et al. [22] combined SII and MLR to predict disease progression and survival. They concluded that using both high SII and high MLR were significantly more likely to predict non-organ-confined disease and poor survival outcomes than single indicator use. Zheng et al. [23] used the combination of simultaneous SII and prognostic nutritional index and showed a powerful independent risk factor for overall survival, CSS, and recurrence-free survival. The combination had the largest area under the curve compared to that for SII or $\mathrm{PNI}$ alone and other clinical factors, indicating its advantage in predicting survival. A large multi-institutional cohort from the international UTUC collaboration study that included 2492 cases with RNU showed that altered SIl was significantly associated with higher pathologic stages and worse survival outcomes [6]. Additionally, the decision curve analysis was used to evaluate the clinical benefit of SII. However, the preoperative SII appears to have relatively limited incremental additive values for clinical decision-making. Unlike the previous two studies $[22,23]$ that combined other parameters, our results were based on the SII alone and show value in providing insight into preoperative prognostic prediction (Supplementary Table 1). Moreover, we demonstrated the SII not only reflects survival prediction but also pathologic surgical outcomes which is more relevant to clinical practice [6].

Laparoscopic RNU has recently emerged as a safe and minimally invasive procedure. However, the oncologic efficacy remains controversial [25-29], especially for locally advanced (T3/T4) UTUC. An European Association of Urology Guidelines Systematic Review [28] collected 42 studies which accounted for 7554 patients: 4925 in the open groups and 2629 in the laparoscopic groups. The results showed that the outcomes of laparoscopic approach may be poorer than those of open methods, especially when dealing the bladder cuff removal. Open RNU may also be much effective in patients with locally advanced (T3/T4) or high-grade tumors. On the other hand, one updated meta-analysis collected eighteen articles with a total of 10,730 participants in the selected papers [29]. They suggest that two different approaches have comparable oncological outcomes in patients with UTUC, even in those with locally advanced disease. We previously compared the oncologic outcomes following RNU using three different methods, intravesical incision, extravesical incision, and transurethral incision (TUI), of managing the bladder cuff [30]. Our results showed no differences in bladder recurrence and cancer-specific survival among the three groups. We also demonstrated no MFS, CSS, and BRFS difference between open and laparoscopic approaches in the current study.

This study had several limitations. First, our results were based on a single-center experience. Second, different surgeons may have their own preferences regarding the treatment decision. Third, we could not exclude all comorbidities that potentially contributed to the poor prognosis. Future larger studies are warranted to compare our findings.

\section{Conclusion}

We found that high preoperative NLR, PLR, and SIl were independent predictive factors for both MFS and CSS in UTUC patients. Among the factors, only elevated SIl can predict bladder recurrence. Therefore, close bladder monitoring during follow-up is recommended.

\section{Abbreviations}

BRFS: Bladder recurrence-free survival; CKD: Chronic kidney disease; CSS: Cancer-specific survival; CT: Computed tomography; eGFR: Estimated glomerular filtration rate; IFN-Y: Interferon gamma; MFS: Metastasis-free survival; NLR: Neutrophile-lymphocyte ratio; PLR: Platelet-lymphocyte ratio; RNU: Radical nephroureterectomy; ROC: Receiver operating characteristic; SII: Systemic immune-inflammation index; TNF-a: Tumor necrosis factor alpha; UBUC: Urinary bladder urothelial carcinoma; UC: Urothelial carcinoma; UTUC: upper tract urothelial carcinoma

\section{Declarations}

\section{Acknowledgement}

Thanks for all co-author and participants.

\section{Authors' contributions}

Dr. Chang and Wu had full access to all the data in the study and takes responsibility for the integrity of the data and the accuracy of the data analysis. TsuMing Chien: data analysis and manuscript writing; Ching-Chia Li: manuscript editing; Yen-Man Lu: manuscript editing; Yii-Her Chou: manuscript editing; HsuehWei Chang: data analysis; Wen-Jeng Wu: project development and manuscript editing. The authors read and approved the final manuscript.

Funding 
Open access funding provided by Kaohsiung Medical University.

\section{Availability of data and materials}

The data used to support the findings of this study are available from the corresponding author upon request.

\section{Ethical approval and consent to participate}

This study was carried out in accordance with the ethical standards of the Helsinki Declaration and approved by the review board of our institution (KMUHIRB-20120138).

\section{Consent for publication}

Consent for publication was obtained from all co-author.

\section{Competing interests}

We declare no competing interests.

\section{References}

1. Siegel RL, Miller KD, Jemal A. Cancer statistics, 2019. CA Cancer J Clin. 2019; 69(1):7-34. doi: 10.3322/caac.21551.

2. Rouprêt M, Babjuk M, Burger M, Capoun O, Cohen D, Compérat EM, et al. European Association of Urology Guidelines on Upper Urinary Tract Urothelial Carcinoma: 2020 Update. Eur Urol. 2021;79(1):62-79. doi: 10.1016/j.eururo.2020.05.042.

3. Margulis V, Shariat SF, Matin SF, Kamat AM, Zigeuner R, Kikuchi E, et al. Upper Tract Urothelial Carcinoma CollaborationThe Upper Tract Urothelial Carcinoma Collaboration. Outcomes of radical nephroureterectomy: a series from the Upper Tract Urothelial Carcinoma Collaboration. Cancer. 2009;115(6):1224-33. doi: 10.1002/cncr.24135.

4. Chien TM, Chan TC, Huang SK, Yeh BW, Li WM, Huang CN, et al. Role of Microtubule-Associated Protein 1b in Urothelial Carcinoma: Overexpression Predicts Poor Prognosis. Cancers (Basel). 2020;12(3):630. doi: 10.3390/cancers12030630.

5. Yu LC, Chang CH, Huang CP, Huang CY, Hong JH, Tai TY, et al. Prognostic Significance of Primary Tumor Location in Upper Tract Urothelial Carcinoma Treated with Nephroureterectomy: A Retrospective, Multi-Center Cohort Study in Taiwan. J Clin Med. 2020;9(12):3866. doi: 10.3390/jcm9123866.

6. Mori K, Resch I, Miura N, Laukhtina E, Schuettfort VM, Pradere B, et al. Prognostic role of the systemic immune-inflammation index in upper tract urothelial carcinoma treated with radical nephroureterectomy: results from a large multicenter international collaboration. Cancer Immunol Immunother. 2021;70(9):2641-2650. doi: 10.1007/s00262-021-02884-w.

7. De Lorenzis E, Albo G, Longo F, Bebi C, Boeri L, Montanari E. Current Knowledge on Genomic Profiling of Upper Tract Urothelial Carcinoma. Genes (Basel). 2021;12(3):333. doi: 10.3390/genes12030333.

8. Robinson BD, Vlachostergios PJ, Bhinder B, Liu W, Li K, Moss TJ, et al. Upper tract urothelial carcinoma has a luminal-papillary T-cell depleted contexture and activated FGFR3 signaling. Nat Commun. 2019;10(1):2977. doi: 10.1038/s41467-019-10873-y.

9. Janisch F, Shariat SF, Baltzer P, Fajkovic H, Kimura S, Iwata T, et al. Diagnostic performance of multidetector computed tomographic (MDCTU) in upper tract urothelial carcinoma (UTUC): a systematic review and meta-analysis. World J Urol. 2020;38(5):1165-1175. doi: 10.1007/s00345-019-02875-8.

10. Grivennikov SI, Greten FR, Karin M. Immunity, inflammation, and cancer. Cell. 2010;140(6):883-99. doi: 10.1016/j.cell.2010.01.025

11. Gakis G, Todenhöfer T, Stenzl A. The prognostic value of hematological and systemic inflammatory disorders in invasive bladder cancer. Curr Opin Urol. 2011;21(5):428-33. doi: 10.1097/MOU.0b013e32834966fa.

12. Vartolomei MD, Kimura S, Ferro M, Vartolomei L, Foerster B, Abufaraj M, et al. Is neutrophil-to-lymphocytes ratio a clinical relevant preoperative biomarker in upper tract urothelial carcinoma? A meta-analysis of 4385 patients. World J Urol. 2018;36(7):1019-1029. doi: 10.1007/s00345-018-2235-5.

13. Bao Y, Wang Y, Li X, Pan M, Zhang H, Cheng Z, et al. Prognostic significance of platelet-to-lymphocyte ratio in urothelial carcinoma patients: a metaanalysis. Cancer Cell Int. 2019;19:315. doi: 10.1186/s12935-019-1032-6.

14. Shao Y, Li W, Wang D, Wu B. Prognostic value of preoperative lymphocyte-related systemic inflammatory biomarkers in upper tract urothelial carcinoma patients treated with radical nephroureterectomy: a systematic review and meta-analysis. World J Surg Oncol. 2020;18(1):273. doi: 10.1186/s12957-02002048-7.

15. Huang Y, Gao Y, Wu Y, Lin H. Prognostic value of systemic immune-inflammation index in patients with urologic cancers: a meta-analysis. Cancer Cell Int. 2020;20:499. doi: 10.1186/s12935-020-01590-4.

16. Li N. Platelets in cancer metastasis: to help the "villain" to do evil. Int J Cancer. 2016;138(9):2078-2087. doi: 10.1002/ijc.29847.

17. Schlesinger M. Role of platelets and platelet receptors in cancer metastasis. J Hematol Oncol. 2018;11(1):125. doi: 10.1186/s13045-018-0669-2.

18. Masucci MT, Minopoli M, Carriero MV. Tumor Associated Neutrophils. Their Role in Tumorigenesis, Metastasis, Prognosis and Therapy. Front Oncol. 2019;9:1146. doi: 10.3389/fonc.2019.01146

19. Wu L, Saxena S, Awaji M, Singh RK. Tumor-Associated Neutrophils in Cancer: Going Pro. Cancers (Basel). 2019;11(4):564. doi: 10.3390/cancers11040564.

20. Zhao J, Huang W, Wu Y, Luo Y, Wu B, Cheng J, et al. Prognostic role of pretreatment blood lymphocyte count in patients with solid tumors: a systematic review and meta-analysis. Cancer Cell Int. 2020;20:15. doi: 10.1186/s12935-020-1094-5.

Page $10 / 12$ 
21. Shen J, Xiao Z, Zhao Q, Li M, Wu X, Zhang L, Hu W, Cho CH. Anti-cancer therapy with TNFa and IFNy: A comprehensive review. Cell Prolif. 2018 Aug;51(4):e12441. doi: 10.1111/cpr.12441.

22. Jan $\mathrm{HC}$, Yang WH, Ou CH. Combination of the Preoperative Systemic Immune-Inflammation Index and Monocyte-Lymphocyte Ratio as a Novel Prognostic Factor in Patients with Upper-Tract Urothelial Carcinoma. Ann Surg Oncol. 2019;26(2):669-684. doi: 10.1245/s10434-018-6942-3.

23. Zheng Y, Yu D, Yu Z, Zhao D, Chen Y, Chen W, et al. Association of preoperative systemic Immune-inflammation Index and Prognostic Nutritional Index with survival in patients with Upper Tract Urothelial Carcinoma. J Cancer. 2020;11(19):5665-5677. doi: 10.7150/jca.44915.

24. Levey AS, Stevens LA, Schmid CH, Zhang YL, Castro AF 3rd, Feldman HI, et al. A new equation to estimate glomerular filtration rate. Ann Intern Med. 2009;150(9):604-12. doi: 10.7326/0003-4819-150-9-200905050-00006.

25. Micali S, Celia A, Bove P, De Stefani S, Sighinolfi MC, Kavoussi LR, et al. Tumor seeding in urological laparoscopy: an international survey. J Urol. 2004;171(6 Pt 1):2151-4. doi: 10.1097/01.ju.0000124929.05706.6b.

26. Xylinas E, Kluth L, Passoni N, Trinh QD, Rieken M, Lee RK, et al. Prediction of intravesical recurrence after radical nephroureterectomy: development of a clinical decision-making tool. Eur Urol. 2014;65(3):650-8. doi: 10.1016/j.eururo.2013.09.003.

27. Liu F, Guo W, Zhou X, Ding Y, Ma Y, Hou Y, et al. Laparoscopic versus open nephroureterectomy for upper urinary tract urothelial carcinoma: A systematic review and meta-analysis. Medicine (Baltimore). 2018;97(35):e11954. doi: 10.1097/MD.0000000000011954.

28. Peyronnet B, Seisen T, Dominguez-Escrig JL, Bruins HM, Yuan CY, Lam T, et al. Oncological Outcomes of Laparoscopic Nephroureterectomy Versus Open Radical Nephroureterectomy for Upper Tract Urothelial Carcinoma: An European Association of Urology Guidelines Systematic Review. Eur Urol Focus. 2019;5(2):205-223. doi: 10.1016/j.euf.2017.10.003.

29. Piszczek R, Nowak Ł, Krajewski W, Chorbińska J, Poletajew S, Moschini M, et al. Oncological outcomes of laparoscopic versus open nephroureterectomy for the treatment of upper tract urothelial carcinoma: an updated meta-analysis. World J Surg Oncol. 2021;19(1):129. doi: 10.1186/s12957-021-02236-Z.

30. Li WM, Shen JT, Li CC, Ke HL, Wei YC, Wu WJ, et al. Oncologic outcomes following three different approaches to the distal ureter and bladder cuff in nephroureterectomy for primary upper urinary tract urothelial carcinoma. Eur Urol. 2010;57(6):963-9. doi: 10.1016/j.eururo.2009.12.032.

\section{Figures}
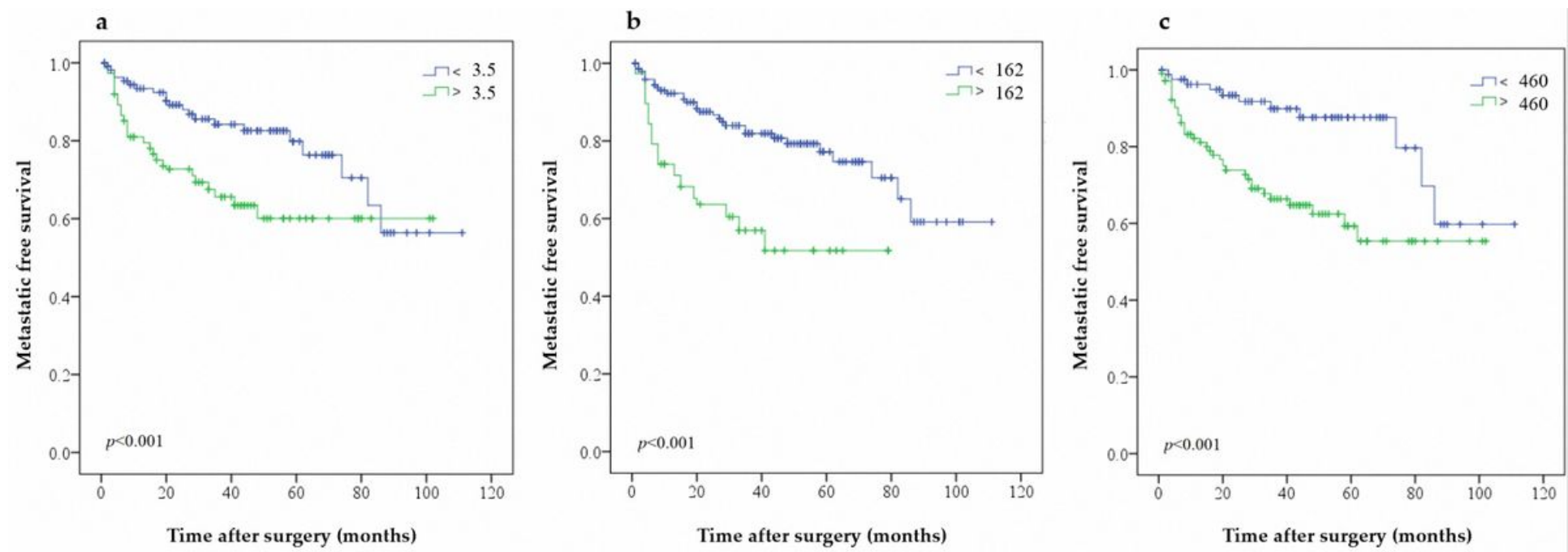

Figure 1

The Kaplan-Meier analysis indicated that patients exhibiting higher NLR, PLR, and SIl showed significantly poor MFS rates (Figures 1a, 1b, and 1c; log-rank, all $P<0.001)$. 

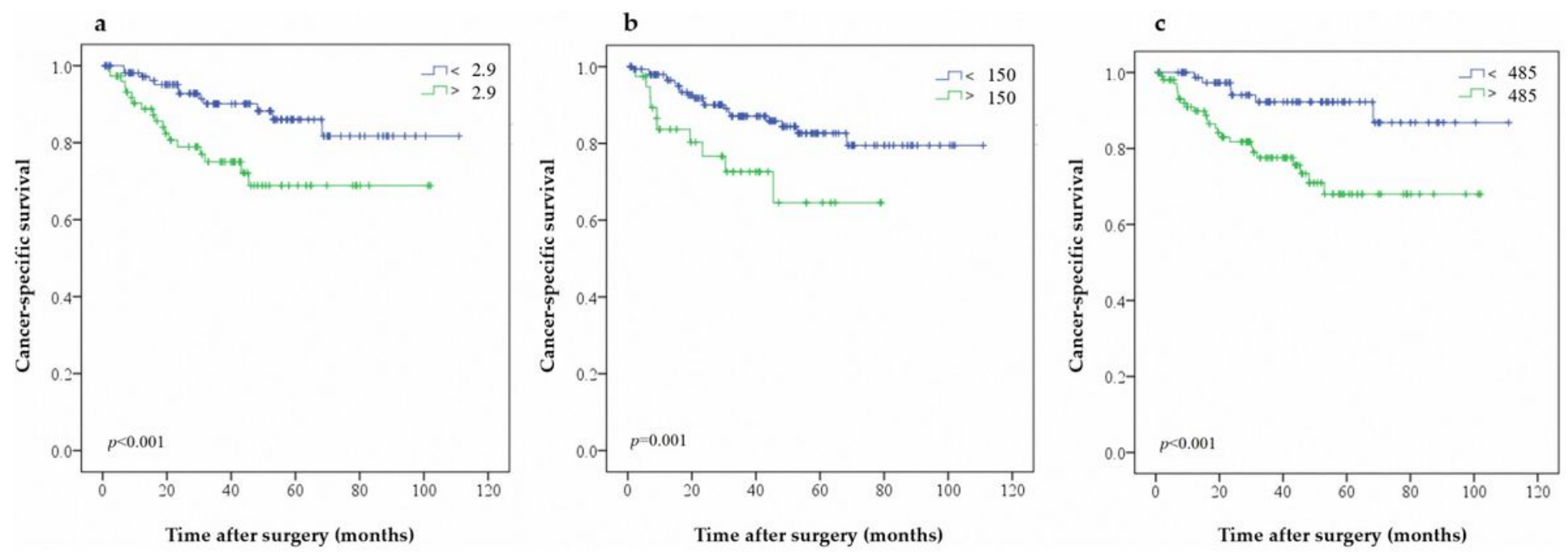

Figure 2

The Kaplan-Meier analysis revealed that patients exhibiting higher NLR, PLR, and SIl showed significantly worse CSS rates
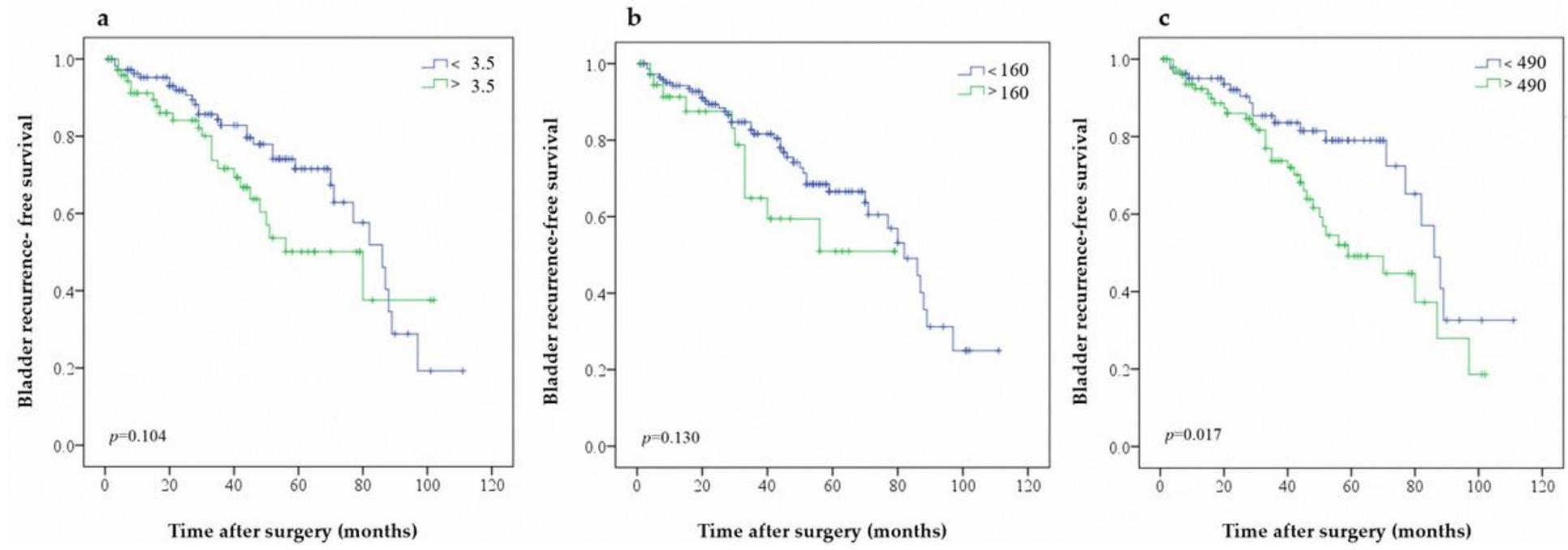

Figure 3

The Kaplan-Meier analysis indicated that patients with higher SII showed significantly worse BRFS rates (Figure 3c). Higher NLR and PLR were not associated with BRFS (Figure 3a, and 3b).

\section{Supplementary Files}

This is a list of supplementary files associated with this preprint. Click to download.

- SupplementaryTable.docx 